Published in "International Journal of Antimicrobial Agents

46(1): 108-110, 2015"

which should be cited to refer to this work.

\title{
Modulation of $m g r B$ gene expression as a source of colistin resistance in Klebsiella oxytoca
}

\author{
Aurélie Jayol ${ }^{\mathrm{a}}$, Laurent Poirel ${ }^{\mathrm{a}, *}$, Maria-Virginia Villegas ${ }^{\mathrm{b}}$, Patrice Nordmann ${ }^{\mathrm{a}, \mathrm{c}}$ \\ a Medical and Molecular Microbiology «Emerging Antibiotic Resistance» Unit, Department of Medicine, Faculty of Science, University of Fribourg, Rue \\ Albert-Gockel 3, $\mathrm{CH}-1700$ Fribourg, Switzerland \\ ${ }^{\mathrm{b}}$ International Center for Medical Research and Training, CIDEIM, Cali, Colombia \\ ${ }^{c}$ Hôpital Fribourgeois - Hôpital Cantonal de Fribourg, Riaz, Switzerland
}

\begin{abstract}
Gene modifications in the PmrAB and PhoPQ two-component regulatory systems, as well as inactivation of the mgrB gene, are known to be causes of colistin resistance in Klebsiella pneumoniae. The objective of this study was to characterise the mechanism involved in colistin resistance in a Klebsiella oxytoca isolate. A K. oxytoca clinical isolate showing resistance to colistin was recovered in Cali, Colombia. The pmrA, pmrB, phoP, phoQ and $m g r B$ genes were amplified and sequenced. Wild-type $m g r B$ genes from $K$. pneumoniae and $K$. oxytoca were cloned, and corresponding recombinant plasmids were used for complementation assays. By analysing the $m g r B$ gene of the $K$. oxytoca isolate and its flanking sequences, an insertion sequence (IS) of $1196 \mathrm{bp}$ was identified in its promoter region. The insertion was located between nucleotides -39 and -38 when referring to the start codon of the $m g r B$ gene, thus negatively interfering with expression of the $\mathrm{mgrB}$ gene by modifying its promoter structure. This IS was very similar to ISKpn26 (99\% nucleotide identity) belonging to the IS5 family. Complementation assays with $m g r B$ genes from wild-type K. pneumoniae or K. oxytoca restored full susceptibility to colistin. In conclusion, here we identified the mechanism involved in colistin resistance in a K. oxytoca isolate. Modulation of mgrB gene expression was the key factor for this acquired resistance to colistin.
\end{abstract}

\section{Introduction}

Klebsiella oxytoca and Klebsiella pneumoniae are frequent sources of nosocomial infections and the sources of nosocomial outbreaks [1,2]. The occurrence of multidrug resistance is increasingly observed among the Gram-negative pathogens Acinetobacter baumannii, Pseudomonas aeruginosa and Enterobacteriaceae. Consequently, use of colistin, a polymyxin-type antibiotic, is being reconsidered in particular for treating critically ill patients infected with these pathogens [3-5]. Unfortunately, the increasing usage of colistin is being associated with the emergence of colistin resistance, in particular in K. pneumoniae [6-11].

Alterations in the two-component regulatory systems PmrAB and PhoPQ $[8,9,12]$ are known to be involved in polymyxin resistance in $K$. pneumoniae. Inactivation of the $\operatorname{mgrB}$ gene,

\footnotetext{
* Corresponding author. Tel.: +41263009582.

E-mail address: laurent.poirel@unifr.ch (L. Poirel).
}

encoding a negative feedback regulator of the PhoPQ twocomponent system, is also a common mechanism involved in colistin resistance in $K$. pneumoniae $[10,13]$. The reported alterations in the $m g r B$ gene include insertion of several insertions sequences (IS5-like, IS1F-like, ISKpn13, ISKpn14) at different locations in the coding sequence of $\operatorname{mgrB}$ and in its promoter region. Non-silent point mutations, premature stop codons, small intragenic deletions and large deletion of the $\mathrm{mgrB}$ locus are also known. These alterations result in either reduced or even lack of expression of the $m g r B$ gene leading to upregulation of the phoPQ and pmrHFIJKLM operons that confer resistance to colistin.

Lippa and Goulian have identified MgrB homologues in the genome sequences of various enterobacterial species [14]. Therefore, alterations in $\operatorname{mgrB}$ gene expression might possibly be involved in acquisition of colistin resistance in different Klebsiella species.

The aim of this study was to identify the mechanism responsible for resistance to colistin in a $K$. oxytoca clinical isolate. 


\section{Materials and methods}

\subsection{Bacterial strain}

The colistin-resistant $K$. oxytoca clinical isolate was identified using an API20E system and the matrix-assisted laser desorption/ionisation time-of-flight (MALDI-TOF) technique ( $\mathrm{AB}$ bioMérieux, La Balme-les-Grottes, France). A colistin-susceptible K. oxytoca clinical isolate was used as a wild-type control.

\subsection{Antimicrobial susceptibility}

Antimicrobial susceptibility testing was performed using Etest strips (AB bioMérieux) on Mueller-Hinton agar plates (Bio-Rad, Marnes-la-Coquette, France) with a 0.5 McFarland inoculum. Minimum inhibitory concentrations (MICs) were interpreted as indicated by the European Committee on Antimicrobial Susceptibility Testing (EUCAST) guidelines (Breakpoint tables for interpretation of MICs and zone diameters. Version 4.0, 2014; http://www.eucast.org). Isolates with colistin MICs of $\leq 2 \mu \mathrm{g} / \mathrm{mL}$ were categorised as susceptible whereas those with MICs of $>2 \mu \mathrm{g} / \mathrm{mL}$ were resistant.

\subsection{PCR amplification and sequencing}

Whole-cell DNA was extracted using a QIAquick Kit (QIAGEN, Courtaboeuf, France) according to the manufacturer's instructions. The entire $p m r A, p m r B$, phoP, phoQ and $m g r B$ genes were amplified with specific oligonucleotides primers (Table 1) designed with sequences of K. oxytoca KCTC 1686 and E718 genomes available on the National Center for Biotechnology Information (NCBI) database (http://www.ncbi.nlm.nih.gov.gate2.inist.fr/). Amplified DNA fragments were purified with a QIAquick PCR Purification Kit (QIAGEN). Both strands of the amplification products obtained were sequenced with an ABI 3100 Sequencer (Applied Biosystems, Foster City, CA). The nucleotide and deduced protein sequences were analysed at the NCBI website (http://www.ncbi.nlm.nih.gov) by the Basic Local Alignment Search Tool (BLAST) programme. The insertion sequence (IS) was analysed using the IS finder website (http:// www-is.biotoul.fr).

\subsection{Complementation assays}

The wild-type $m g r B$ gene from a colistin-susceptible $K$. oxytoca strain and a $K$. pneumoniae strain, as well as the non-coding $m d h$ sequence [15], were amplified by PCR using 2X Phusion ${ }^{\circledR}$ HF Master Mix (Finnzymes; Life Technologies, Illkirch, France) and the primers listed in Table 1 . The amplified fragments were cloned into the plasmid $\mathrm{pCR}^{\circledR}$-Blunt II-TOPO ${ }^{\circledR}$ (Invitrogen, Illkirch, France)

Table 1

Primers used in this study.

\begin{tabular}{|c|c|c|c|}
\hline Primer & Sequence $\left(5^{\prime}-3^{\prime}\right)$ & Gene & Ref. \\
\hline Kox pmrC ext F & GCC GAT ATC GCA GGG GTT AA & pmrC & This study \\
\hline Kox pmrC ext $\mathrm{R}$ & TAA CAG GAG CGC ATC GTC TT & pmrC & \\
\hline Kox pmrA ext $\mathrm{F}$ & GCA AGC GTA TTC GCA GGA TA & pmrA & \\
\hline Kox pmrA ext $\mathrm{R}$ & CTG ATG AGC TGA CAA ACG GC & pmrA & \\
\hline Kox pmrB ext F & CAA CGA CAT TTA CAG CTG GG & pmrB & \\
\hline Kox pmrB ext $\mathrm{R}$ & CTT TAC TGA GGA TAG CGC CA & pmrB & \\
\hline Kox phoP ext F & CCA AGA GAC CGA GGT ACA AA & phoP & \\
\hline Kox phoP ext R & GAG TGA CAA CAC CAG CAC TA & phoP & \\
\hline Kox phoQ ext F & CCA TAC CAT CGA TGT GCT GA & phoQ & \\
\hline Kox phoQ ext R & GCA GGT GTC TGT TAG GGA TT & phoQ & \\
\hline Kox mgrB ext $\mathrm{F}$ & CGC GGT TTA AGA AGG TCA TG & $m g r B$ & \\
\hline Kox mgrB ext $\mathrm{R}$ & AGG CGT TTA TTC TAC CAC CC & $\operatorname{mgrB}$ & \\
\hline mdh F & CCC AAC TCG CTT CAG GTT CAG & $m d h$ & [15] \\
\hline mdh R & CCG TTT TTC CCC AGC AGC AG & $m d h$ & \\
\hline
\end{tabular}

and the resulting plasmids pTOPO-mgrBKox, pTOPO-mgrBKp and pTOPO- $m d h$ (encoding resistance to Zeocin ${ }^{\mathrm{TM}}$ ) were respectively transformed into an electrocompetent colistin-resistant $K$. oxytoca isolate by electroporation. Electrotransformants were selected by overnight incubation at $37^{\circ} \mathrm{C}$ on Mueller-Hinton agar supplemented with $100 \mu \mathrm{g} / \mathrm{mLZeocin}^{\mathrm{TM}}$. MICs of colistin for the $K$. oxytoca transformants were determined by Etest (bioMérieux).

\section{Results and discussion}

\subsection{Strain and patient features}

The colistin-resistant $K$. oxytoca isolate C24 was recovered in February 2008 from a soft-tissue secretion from a 66-yearold male patient hospitalised for an intra-abdominal infection in Cali, Colombia. The isolate was resistant to broad-spectrum cephalosporins (MICs of ceftazidime and cefotaxime $=32 \mu \mathrm{g} / \mathrm{mL}$ ), amikacin ( $\mathrm{MIC}=16 \mu \mathrm{g} / \mathrm{mL}$ ) and colistin ( $\mathrm{MIC}=24 \mu \mathrm{g} / \mathrm{mL}$ ) and was of intermediate susceptibility to ciprofloxacin (MIC $=1 \mu \mathrm{g} / \mathrm{mL}$ ). The isolate was susceptible to carbapenems (MIC $<0.5 \mu \mathrm{g} / \mathrm{mL}$ ), gentamicin ( $\mathrm{MIC}=0.5 \mu \mathrm{g} / \mathrm{mL}$ ) and tigecycline $(\mathrm{MIC}=0.5 \mu \mathrm{g} / \mathrm{mL})$. It harboured a bla $a_{\mathrm{CTX}-\mathrm{M}-15}$ gene but did not produce any carbapenemase according to the Carba NP test. To our knowledge, the patient had never been treated with any polymyxin.

\subsection{Modification of mgrB gene expression through insertion of an insertion sequence element}

Sequence analysis of the $p m r A, p m r B$, phoP and phoQ genes known to be involved in lipopolysaccharide (LPS) synthesis showed $100 \%$ identity with the genes identified from colistin-susceptible wild-type $K$. oxytoca isolates (data not shown). Sequence analysis of the $m g r B$ gene as well as upstream-located sequences revealed that an IS of $1196 \mathrm{bp}$ had targeted the upstream vicinity of the $m g r B$ gene between nucleotides -38 and -39 when referring to the start codon of $m g r B$. This IS was similar to ISKpn26 (differing by only $5 \mathrm{bp}$ ) and belonged to the IS5 family. It was bracketed by a 4-bp duplication (TTAT) being the likely signature of a transposition process. The origin of ISKpn26 is K. pneumoniae (https://www-is.biotoul.fr/index. html?is_special_name=ISKpn26) and it has also been identified in K. oxytoca KONIH1 (GenBank accession no. CP008788.1). In silico analysis of the sequence located upstream of a wild-type $m g r B$ gene revealed a putative promoter sequence made of -35 [TTGAAA] and -10 [TAAACT] boxes separated by $15 \mathrm{bp}$ (Fig. 1). Insertion of ISKpn26 in isolate C24 led to displacement of this promoter with respect to the $m g r B$ gene position, the corresponding -35 and -10 sequences being consequently very distant from it (Fig. 1). This suggests a decrease or even lack of expression of the $m g r B$ gene, leading to low or absence of production of the corresponding protein and consequently to acquired resistance to colistin.

\subsection{Complementation experiments}

To confirm whether higher expression of $m g r B$ might restore susceptibility to colistin, complementation of isolate C24 with a plasmid overexpressing $m g r B$ was performed. Transformation assays were either performed with plasmids encoding the MgrB protein of $K$. oxytoca or $K$. pneumoniae, respectively. The $m g r B$ gene was provided in trans together with its original promoter in order to ensure it would be expressed at a wild-type level. Following complementation, isolate C24 recovered full susceptibility to colistin with the wild-type MgrB of $K$. oxytoca $(\mathrm{MIC}=0.047 \mu \mathrm{g} / \mathrm{mL}$ ). Interestingly, the same MIC was obtained after complementation with the MgrB of $K$. pneumoniae ( $\mathrm{MIC}=0.047 \mu \mathrm{g} / \mathrm{mL}$ ). As expected, 


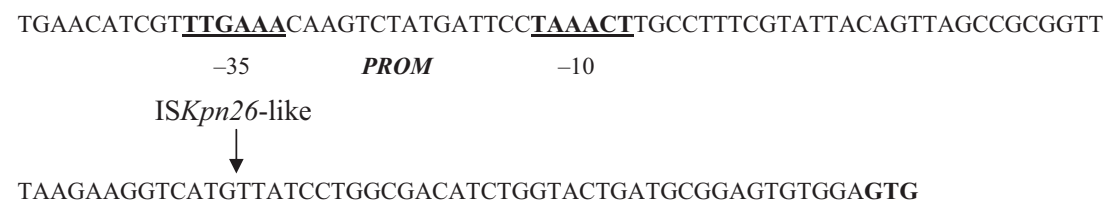

$\rightarrow \operatorname{mgrB}$ start codon

Fig. 1. Sequences located at the $5^{\prime}$-extremity of the $m g r B$ gene. The putative promoter $(P R O M)$ is indicated, with its respective -35 and -10 boxes underlined. The arrow indicates the target site for insertion of the insertion sequence ISKpn26-like. The mgrB start codon is in bold.

transformation with plasmid pTOPO-mdh used as a negative control did not restore susceptibility to colistin (MIC $=24 \mu \mathrm{g} / \mathrm{mL}$ ).

3.4. Comparison of mgrB gene sequences of $\mathrm{K}$. oxytoca and $\mathrm{K}$. pneumonia

Alignment of the $\operatorname{mgrB}$ sequences from wild-type K. pneumoniae MGH78578 and K. oxytoca KCTC1686 strains showed that the sequences were almost identical [only a single amino acid substitution (Cys to Tyr at amino acid position 28)]. This result explains why complementation with the $\mathrm{mgrB}$ gene of $\mathrm{K}$. pneumoniae restored full susceptibility to colistin in the $K$. oxytoca isolate. Furthermore, it may suggest that all previously reported alterations of the $m g r B$ gene $[10,13]$ that have been shown to be the sources of acquired resistance to colistin in K. pneumoniae (such as insertions of IS elements at different locations in the coding sequence of $m g r B$, non-silent point mutations, premature stop codons, intragenic deletions, etc.) might also be a source of colistin resistance in K. oxytoca.

\section{Conclusion}

This study reports on the molecular identification of colistin resistance in $K$. oxytoca. This resistance mechanism is associated with modification of the biosynthesis pathway of LPS. It is similar to that reported in K. pneumoniae, underlining that common mechanisms of colistin resistance could be identified in different enterobacterial species.

\section{Nucleotide sequence accession number}

The nucleotide sequences of the mutated $m g r B$ genes identified in this study have been deposited at DDBJ/EMBL/GenBank under accession no. KP748172.

\section{Funding}

This work was funded by the University of Fribourg (Fribourg, Switzerland) and by a grant from the European Community [MAGIC-BULLET, FP7/HEALTH-F3-2001-278232].

\section{Competing interests}

None declared.

\section{Ethical approval}

Not required.

\section{References}

[1] Hoenigl M, Valentin T, Zarfel G, Wuerstl B, Leitner E, Salzer HJ, et al. Nosocomial outbreak of Klebsiella pneumoniae carbapenemase-producing Klebsiella oxytoca in Austria. Antimicrob Agents Chemother 2012;56:2158-61.

[2] Zarate MS, Gales AC, Picao RC, Pujol GS, Lanza A, Smayevsky J. Outbreak of OXY-2-producing Klebsiella oxytoca in a renal transplant unit. J Clin Microbiol 2008;46:2099-101.

[3] Falagas ME, Rafailidis PI, Ioannidou E, Alexiou VG, Matthaiou DK, Karageorgopoulos DE, et al. Colistin therapy for microbiologically documented multidrug-resistant Gram-negative bacterial infections: a retrospective cohort study of 258 patients. Int J Antimicrob Agents 2010;35:194-9.

[4] Landman D, Georgescu C, Martin DA, Quale J. Polymyxins revisited. Clin Microbiol Rev 2008;21:449-65.

[5] Li J, Nation RL, Owen RJ, Wong S, Spelman D, Franklin C. Antibiograms of multidrug-resistant clinical Acinetobacter baumannii: promising therapeutic options for treatment of infection with colistin-resistant strains. Clin Infect Dis 2007;45:594-8.

[6] Ah YM, Kim AJ, Lee JY. Colistin resistance in Klebsiella pneumoniae. Int J Antimicrob Agents 2014;44:8-15.

[7] Cannatelli A, D'Andrea MM, Giani T, Di Pilato V, Arena F, Ambretti S, et al. In vivo emergence of colistin resistance in Klebsiella pneumoniae producing KPC-type carbapenemase mediated by insertional inactivation of the PhoQ/PhoP mgrB regulator. Antimicrob Agents Chemother 2013;57:5521-6.

[8] Cannatelli A, Di Pilato V, Giani T, Arena F, Ambretti S, Gaibani P, et al. In vivo evolution to colistin resistance by PmrB sensor kinase mutation in KPC-producing Klebsiella pneumoniae is associated with low-dosage colistin treatment. Antimicrob Agents Chemother 2014;58:4399-403.

[9] Jayol A, Poirel L, Brink A, Villegas MV, Yilmaz M, Nordmann P. Resistance to colistin associated with a single amino acid change in protein PmrB among Klebsiella pneumoniae isolates of worldwide origin. Antimicrob Agents Chemother 2014;58:4762-6.

[10] Poirel L, Jayol A, Bontron S, Villegas MV, Özdamar M, Türkoglu S, et al. The mgrB gene as a key target for acquired resistance to colistin in Klebsiella pneumoniae. J Antimicrob Chemother 2015:70:75-80.

[11] Monaco M, Giani T, Raffone M, Arena F, Garcia-Fernandez A, Pollini S, et al. Colistin resistance superimposed to endemic carbapenem-resistant Klebsiella pneumoniae: a rapidly evolving problem in Italy, November 2013 to April 2014 Euro Surveill 2014;19, pii:20939.

[12] Cheng HY, Chen YF, Peng HL. Molecular characterization of the PhoPQ-PmrD-PmrAB mediated pathway regulating polymyxin B resistance in Klebsiella pneumoniae CG43. J Biomed Sci 2010;17:60.

[13] Cannatelli A, Giani T, D'Andrea MM, Di Pilato V, Arena F, Conte V, et al MgrB inactivation is a common mechanism of colistin resistance in KPC carbapenemase-producing Klebsiella pneumoniae of clinical origin. Antimicrob Agents Chemother 2014;58:5696-703.

[14] Lippa AM, Goulian M. Feedback inhibition in the PhoQ/PhoP signaling system by a membrane peptide. PLoS Genet 2009;5:e1000788.

[15] Diancourt L, Passet V, Verhoef J, Grimont PA, Brisse S. Multilocus sequence typing of Klebsiella pneumoniae nosocomial isolates. J Clin Microbiol 2005;43:4178-82. 